\title{
Reinhard Hujer - Ein Forscherleben als Spiegelbild der Ökonometrie
}

\author{
Marco Caliendo $\cdot$ Joachim Grammig $•$ Hilmar Schneider
}

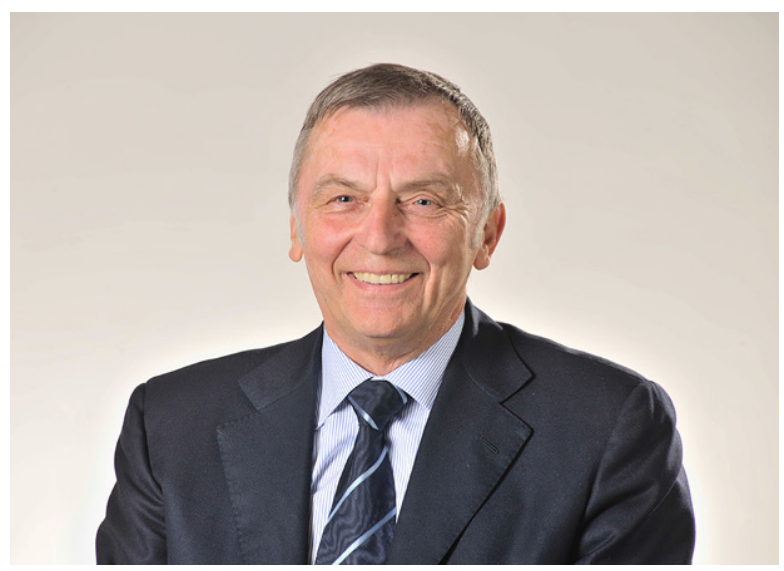

Für alle unerwartet verstarb Professor Dr. Reinhard Hujer am 13. August 2020, wenige Tage vor Vollendung seines 80. Lebensjahres, nach kurzer schwerer Krankheit.

Zeit seines Lebens war er getrieben von der Überzeugung, dass empirisch fundierte Forschung die Grundlage einer rational aufgeklärten Wirtschafts- und Sozialpolitik sein müsse. Er war fasziniert von der Ökonometrie als einem Instrument, mit dem sich die hinter der Vielfalt von Einzelbeobachtungen verborgenen strukturellen Zusammenhänge wie durch ein Mikroskop sichtbar machen lassen. Er bewegte sich dabei stets entlang der Grenzen des technisch Möglichen. Sein Forscherleben ist über

H. Schneider $(\bowtie)$

Forschungsinstitut zur Zukunft der Arbeit (IZA), Schaumburg-Lippe-Str. 5-9, 53113 Bonn,

Deutschland

E-Mail: Schneider@iza.org 
drei Jahrzehnte hinweg ein unmittelbares Spiegelbild der Methodenentwicklung in der Ökonometrie.

Der Beginn seiner wissenschaftlichen Karriere stand noch ganz im Zeichen einer makroökonomisch geprägten Tradition der empirischen Wirtschaftsforschung. Im Sinne Tinbergens ging es ihm darum, empirisch fundierte Grundlagen für eine rational fundierte Planung einer Volkswirtschaft als Systemkreislauf zu schaffen und $\mathrm{zu}$ verstehen.

1974 nahm er den Ruf auf den Lehrstuhl für VWL, Statistik und Ökonometrie an der TU Darmstadt an. In diese Zeit fällt die Veröffentlichung des Lehrbuchs „Grundlagen der empirischen Wirtschaftsforschung“, das er zusammen mit Rolf Cremer verfasste.

1979 folgte dann der Ruf auf den Lehrstuhl für Statistik und Ökonometrie an der Goethe-Universität Frankfurt am Main, den er bis zu seiner Emeritierung im Jahr 2005 inne hatte. Die Goethe-Universität hatte sich damals gerade erfolgreich zusammen mit der Universität Mannheim auf die Einrichtung eines neuen Sonderforschungsbereichs ( $\mathrm{Sfb}$ ) beworben. Mit dem Sfb 3 hatte die Deutsche Forschungsgemeinschaft (DFG) erstmals einen Sonderforschungsbereich in den Wirtschaftsund Sozialwissenschaften bewilligt, der sich vom Finanzvolumen her mit den Großforschungsprojekten in den Naturwissenschaften messen konnte. Damit bot sich Reinhard Hujer eine Chance, die für sein ganzes späteres akademisches Leben prägend gewesen sein dürfte.

Das Generalthema des Sonderforschungsbereichs lautete „Mikroanalytische Grundlagen der Gesellschaftspolitik“. Dessen Programm war ein Reflex auf die Einsicht, dass die bis dahin vorherrschende makro-ökonomische Modellierung der Vielfalt struktureller Gegebenheiten und insbesondere Verteilungsaspekten nur ungenügend Rechnung tragen konnte.

Von seinem Lehrstuhlvorgänger Gerd Hansen übernahm Reinhard Hujer zunächst die Leitung des Teilprojekts Makrosimulation. Es umfasste die Weiterentwicklung des sogenannten Frankfurter Modells, das mit seinen gut 500 Einzelgleichungen zur Beschreibung makroökonomischer Zusammenhänge für damalige Verhältnisse enorm komplex war. Das Frankfurter Modell war dazu gedacht, die Grundlage für eine mittelfristig geplante Verschmelzung von Mikro- und Makromodellierung zu legen. Zwar erwies sich dieses Ziel für die damaligen Möglichkeiten letztlich als zu ambitioniert, gleichwohl wurden unschätzbar wichtige Grundlagen für den Modellierungsfortschritt gelegt. Reinhard Hujer war dafür eine treibende Kraft.

Seine fachliche Kompetenz und sein strategischer Weitblick, aber auch sein ausgleichendes Wesen brachten ihm rasch eine Führungsrolle in diesem Forschungsverbund ein. Seine Erfolgsgeschichte verdankt der Sfb 3 neben seinen Gründervätern Richard Hauser, Hans-Jürgen Krupp und Wolfgang Zapf auch ganz maßgeblich seinem späteren Sprecher Reinhard Hujer.

Eine der Pionierleistungen des Sonderforschungsbereichs war die Etablierung des Sozio-oekonomischen Panels, das bis heute die Informationsquelle für die Forschung zur sozio-ökonomischen Lage der Haushalte in Deutschland darstellt. Schon die 1984 erhobene erste Befragungswelle enthielt ein retrospektives Kalendarium zu individuellen Einkommensverläufen und Erwerbsstatus. Damit war eine Datengrundlage geschaffen, wie es sie zumindest in Deutschland noch nie zuvor gegeben 
hatte. Längsschnittdaten eröffneten erstmals die Möglichkeit, den Einfluss unbeobachteter Heterogenität auf die Schätzung von strukturellen Parametern zu kontrollieren und damit systematische Schätzfehler zu korrigieren, die bei der bis dahin üblichen Verwendung von Querschnittsdaten oder aggregierten Daten unvermeidlich waren.

Es ist und bleibt bemerkenswert, dass gerade ein überzeugter Makro-Ökonometriker wie Reinhard Hujer die daraus resultierenden Möglichkeiten als einer der ersten vorhergesehen und entschlossen genutzt hat. Das Teilprojekt Makrosimulation wurde kurzerhand um den Baustein Panelökonometrie erweitert. Was anfangs noch wie ein Fremdkörper wirkte, avancierte in der Folge zum beherrschenden Schwerpunkt. Diese Phase in den 1980er-Jahren markiert den Wandel vom Makro- zum MikroÖkonometriker, wie ihn wohl kaum jemand anders in der Profession vollzogen haben dürfte.

Die Standards der ökonometrischen Welt wurden damals in den USA gesetzt. Reinhard Hujer begann damit, die dort entwickelten Methoden zunächst anhand der Literatur zu adaptieren und avancierte mit seinen Publikationen etwa zu den Determinanten der Arbeitslosigkeitsdauer rasch zu einem der führenden Arbeitsmarktforscher in Deutschland. Der Sonderforschungsbereich eröffnete ihm darüber hinaus wichtige internationale Vernetzungsmöglichkeiten. So knüpfte er im Rahmen von zwei Gastprofessuren (1986 und 1989) an der Stanford University aktive Verbindungen zu Größen wie Takeshi Amemya und Tom MaCurdy, die durch weitere Besuche und Gegenbesuche vertieft wurden. Dieser Austausch zündete eine neue Stufe der Forschungsaktivitäten an seinem Lehrstuhl, die sich in zahlreichen Publikationen in einschlägigen Journals niederschlug.

Arbeitsmarktthemen sollten von da an das Markenzeichen von Reinhard Hujer bleiben. Als Mitte der 1990er-Jahre die kausalanalytische Evaluationsforschung ihren Aufschwung nahm, hat Reinhard Hujer ein weiteres wichtiges Forschungsgebiet methodisch, inhaltlich und auch in Bezug auf die Datenverfügbarkeit entscheidend geprägt. Angeregt von den Arbeiten des späteren Nobelpreisträgers James J. Heckman hat er sich zunächst mit den Effekten von Trainings- und Weiterbildungsmaßnahmen beschäftigt und diese hauptsächlich auf Basis von Daten des Sozio-oekonomischen Panels ausgewertet. Neben den von ihm bereits häufig in anderem Kontext verwendeten Verweildauermodellen, hat er dabei vor allem auch den Einsatz von modernsten mikroökonometrischen Matching-Verfahren vorangetrieben. Mit seinen Arbeiten hatte er eine Vorreiterrolle inne und hat Meilensteine für die evidenzbasierte Politikberatung in Deutschland gesetzt, die nicht zuletzt im Rahmen der Evaluation der Hartz-Reformen Anfang der 2000er-Jahre einen ersten Höhepunkt erlebte.

Fast noch wichtiger für die Entwicklung der Evaluationsforschung in Deutschland war seine Pionierarbeit im Bereich der administrativen Datenerschließung für die wissenschaftliche Evaluation. Die Zeichen der Zeit antizipierend - in diesem Fall die Bedeutung der Qualität von hochwertigen Individualdaten - initiierte und leitete Reinhard Hujer ein von der DFG gefördertes Projekt zur Erschließung administrativer Daten der Bundesagentur für Arbeit (BA) in Zusammenarbeit mit dem Institut für Arbeitsmarkt- und Berufsforschung (IAB) in Nürnberg. Er hat damit maßgebliche Vorarbeit für die heute im Forschungsdatenzentrum des IAB verfügbaren 
Integrierten Erwerbsbiographien geleistet, eine Datengrundlage, von der inzwischen zahlreiche Arbeitsmarktforscher auf der ganzen Welt profitieren.

Mit dem IAB verband ihn übrigens eine langfristige und überaus produktive Kooperation. Aufgrund seiner hervorragenden wissenschaftlichen Verdienste erhielt er 1995 den Ruf auf die Leitungsposition des Instituts. Zwar entschied er sich seinerzeit gegen dieses Angebot, wohl auch, weil er befürchtete, seinem Forscherdrang in einer solchen Funktion nicht mehr in genügendem Maße folgen zu können, aber in der Folgezeit blieb er dem IAB in verschiedenen Funktionen eng verbunden. So war er von 1995 bis 2013 mit kurzer Unterbrechung Mitglied im Herausgebergremium der Zeitschrift für Arbeitsmarktforschung/Journal for Labour Market Research, zeitweise auch als dessen geschäftsführender Herausgeber. Von 2004 bis 2013 gehörte er dem Wissenschaftlichen Beirat des IAB an, davon die letzten fünf Jahre als dessen Vorsitzender.

Gegen Ende seines akademischen Wirkens kehrte Reinhard Hujer in gewisser Weise wieder zu seinen Wurzeln zurück. Als deutlich wurde, dass die mikro-ökonometrisch fundierte Evaluation ihre Grenzen hat, weil sich allgemeine Gleichgewichtseffekte damit nicht ermitteln lassen, wandte er sich den inzwischen entwickelten makroökonometrischen Verfahren zu, mit deren Hilfe sich regionale und gesamtwirtschaftliche Effekte der Arbeitsmarktpolitik evaluieren lassen.

Auch wenn Reinhard Hujer den meisten als bahnbrechender Arbeitsmarktforscher in Erinnerung bleiben wird, dürfen seine Vorstöße in andere Gefilde der Ökonometrie nicht unerwähnt bleiben. Sie waren stets davon geprägt, Bewährtes mit Neuem zu kombinieren und daraus eine fruchtbare Synthese abzuleiten.

Angeregt durch die „Presidential Address“ des späteren Nobelpreisträgers Robert Engle beim Weltkongress der Econometric Society im Jahre 1996, begann Reinhard Hujer die aus der Arbeitsmarktforschung bekannten Verweildauermodelle auf die Analyse der Finanzmarkt-Mikrostruktur zu übertragen. Dabei ging es um die Modellierung der Preisfindung und Liquiditätsdynamik, Themen, die für die Gestaltung von elektronischen Börsenplätzen wie etwa dem Xetra-System der Deutschen Börse von großer Bedeutung sind.

Etwa zeitgleich begann, auf Initiative vor allem von Jan Krahnen, die Finanzwirtschaft (,Finance“) an der Goethe-Universität einen bemerkenswerten Aufschwung zu nehmen, der mit der Einrichtung des Leibniz-Instituts für „Sustainable Financial Architecture" jüngst einen Höhepunkt fand. Reinhard Hujer lieferte mit seiner Forschung einen wichtigen initialen Beitrag für diese Entwicklung, indem er an der Universität Frankfurt das damals aufstrebende Gebiet der Finanzmarktökonometrie etablierte.

Ebenso passend für den Standort Frankfurt, eine Stadt, die den größten kontinentaleuropäischen Flughafen vor der Haustür hat, war, dass Reinhard Hujer mikroökonometrische Modelle auf Probleme der Luftfahrt anwendete. So publizierte er mit Co-Autoren aus seinem Team und der Unternehmensberatung Roland Berger eine Arbeit über ,The Econometrics of Airline Network Management“", in der ein multinomiales Probit-Modell vorgeschlagen wird, das der für die Flugplanung zentralen Problematik der Unabhängigkeit von irrelevanten Alternativen Rechnung trägt.

Auch die Anwendung von mikroökonometrischen Modellen in der Marktforschung fand sein Interesse. So war Reinhard Hujer wissenschaftlich begleitend bei 
der Markteinführung einer Reihe pharmazeutischer Produkte tätig. Mit seinem Team adaptierte er mikroökonometrische Modelle, welche etablierte Methoden des Marketings (die Conjoint-Analyse) ergänzten und so den Weg in die praktische Anwendung fanden.

Als Betreuer war es Reinhard Hujer immer wichtig, seinen wissenschaftlichen Mitarbeiterinnen und Mitarbeitern den Weg in die akademische Welt zu weisen. Dies geschah zum einen natürlich durch die Einbindung in spannende Forschungsprojekte. Zum anderen hat er aber auch von Anfang an dafür gesorgt, dass die Mitarbeiterinnen und Mitarbeiter mit eigenen Beiträgen auf nationalen und vor allem auch internationalen Konferenzen und Workshops wichtige Erfahrungen sammeln konnten. Darüber hinaus hatte er als Mentor immer ein offenes Ohr und stand gerne und engagiert beratend zur Seite. Als Ergebnis dieser Nachwuchsförderung haben heute sieben seiner Schüler Professuren für Ökonometrie, Statistik, empirische Wirtschaftsforschung, angewandte Wirtschaftspolitik und Finanzwissenschaften, aber auch im Bereich der empirischen Betriebswirtschaftslehre inne.

Das Schaffen von Reinhard Hujer hat zahlreiche Spuren hinterlassen und wird noch lange nachwirken. Das gilt nicht nur für die zahlreichen Publikationen, die seinen Namen tragen. Es gilt vor allem für die Inspiration, die er seinen Schülerinnen und Schülern mitgegeben hat, für die wir ihm an dieser Stelle posthum und stellvertretend unseren innigsten Dank aussprechen möchten.

Reinhard Hujer wurde im Kreise seiner Familie und unter Anteilnahme vieler Freunde, Kolleginnen und Kollegen am 24. August 2020 auf dem Alten Friedhof in Darmstadt beigesetzt.

Funding Open Access funding enabled and organized by Projekt DEAL.

Open Access Dieser Artikel wird unter der Creative Commons Namensnennung 4.0 International Lizenz veröffentlicht, welche die Nutzung, Vervielfältigung, Bearbeitung, Verbreitung und Wiedergabe in jeglichem Medium und Format erlaubt, sofern Sie den/die ursprünglichen Autor(en) und die Quelle ordnungsgemäß nennen, einen Link zur Creative Commons Lizenz beifügen und angeben, ob Änderungen vorgenommen wurden.

Die in diesem Artikel enthaltenen Bilder und sonstiges Drittmaterial unterliegen ebenfalls der genannten Creative Commons Lizenz, sofern sich aus der Abbildungslegende nichts anderes ergibt. Sofern das betreffende Material nicht unter der genannten Creative Commons Lizenz steht und die betreffende Handlung nicht nach gesetzlichen Vorschriften erlaubt ist, ist für die oben aufgeführten Weiterverwendungen des Materials die Einwilligung des jeweiligen Rechteinhabers einzuholen.

Weitere Details zur Lizenz entnehmen Sie bitte der Lizenzinformation auf http://creativecommons.org/ licenses/by/4.0/deed.de. 